\title{
Trivium
}

Revue franco-allemande de sciences humaines et sociales - Deutsch-französische Zeitschrift für Geistesund Sozialwissenschaften

$8 \mid 2011$

Traductions et transferts des savoirs dans l'espace euro-méditerranéen à l'époque médiévale

\section{Médecins sans frontières. Médecins melkites, juifs et samaritains en Égypte et en Syrie à l'époque des croisades}

\author{
Johannes Pahlitzsch \\ Traducteur : Mathieu Olivier
}

\section{OpenEdition Journals}

Édition électronique

URL : http://journals.openedition.org/trivium/3962

DOI : 10.4000 /trivium.3962

ISSN : 1963-1820

Éditeur

Les éditions de la Maison des sciences de l'Homme

\section{Référence électronique}

Johannes Pahlitzsch, « Médecins sans frontières. Médecins melkites, juifs et samaritains en Égypte et en Syrie à l'époque des croisades », Trivium [En ligne], 8| 2011, mis en ligne le 16 mai 2011, consulté le 08 septembre 2020. URL : http://journals.openedition.org/trivium/3962 ; DOI : https://doi.org/

10.4000/trivium.3962

Ce document a été généré automatiquement le 8 septembre 2020

\section{(c) $($ ()) $\Theta$}

Les contenus des la revue Trivium sont mis à disposition selon les termes de la Licence Creative Commons Attribution - Pas d'Utilisation Commerciale - Pas de Modification 4.0 International. 


\title{
Médecins sans frontières. Médecins melkites, juifs et samaritains en Égypte et en Syrie à l'époque des croisades
}

\author{
Johannes Pahlitzsch \\ Traduction : Mathieu Olivier
}

\section{NOTE DE L'ÉDITEUR}

Nous remercions Johannes Pahlitzsch et la da maison d'édition Böhlau de nous avoir accordé l'autorisation de traduire cet article dans le présent numéro.

Ibn Ğumay' (mort en 1193), le médecin juif de Saladin, raconte que le sultan, à la faveur d'un moment de répit où il n'était pas accaparé par les affaires du gouvernement, s'entretint avec lui de l'état de la médecine dans son empire. C'est, nous dit Ibn Ğumay', aiguillonné par cette conversation que lui-même entreprit par la suite de rédiger un traité sur ce thème ${ }^{1}$. Ibn Ğumay ${ }^{`}$ divise son œuvre en trois parties. Dans un premier temps, il présente la discipline médicale de façon générale, ses qualités et son utilité ; il insiste ce faisant sur la complexité de la discipline, et, partant, sur la difficulté et la durée de la formation. Dans une seconde partie, l'auteur offre un tableau de l'histoire de la médecine, tableau très clair, car concis et prégnant, à défaut d'être original ; elle est à ses yeux, pour l'essentiel, celle d'une décadence.

Fidèle à l'interprétation transmise par les sources arabes, Ibn Ğumay' décrit la façon dont la science médicale grecque, fondée par Hippocrate et Galien, puis synthétisée par l'École d'Alexandrie, migra à Bagdad au cours du VIII ${ }^{\mathrm{e}}$ siècle. La réception du corpus galénique par les auteurs arabes, qui commença véritablement au $\mathrm{X}^{\mathrm{e}}$ siècle, marqua aux yeux d'Ibn Ğumay' le début d'une nouvelle ère de déclin qui se serait poursuivie jusqu'à son époque. Comme cela avait été le cas à Alexandrie déjà, les 
doctrines de Galien se trouvèrent synthétisées dans de vastes manuels ${ }^{2}$. Mais, au contraire d'Oribase et de ses contemporains, les compilateurs arabes, selon Ibn Ğumay', croyaient pouvoir se substituer aux œuvres des Anciens. À lui seul, le titre de l'ouvrage d'al-Mağūīī, «Somme de l'art médical » (Kāmil aș-șinā'a aț-țibbìya) ${ }^{3}$ trahit pour Ibn Ğumay les prétentions indues de ces médecins. La conséquence en est, poursuit-il, que les étudiants croient qu'il suffit d'avoir lu quelques-uns de ces compendia pour devenir un vrai médecin. Mais comme en réalité ils ne maîtrisent pas les connaissances médicales, ils manient l'esbroufe pour tenter d'impressionner leurs patients ${ }^{4}$.

Dans le troisième et dernier chapitre de son traité, Ibn Ğumay détaille les mesures qui, de son point de vue, seraient indispensables pour améliorer la situation. À ce propos, il renvoie les gouvernants à leurs responsabilités : à eux, en premier lieu, de veiller à ce que seuls les meilleurs professeurs reçoivent la licence d'enseigner; il serait souhaitable qu'ils enseignent uniquement les œuvres de Galien et d'Hippocrate et qu'ils soient en outre en charge de la formation pratique des étudiants, dont le cadre approprié serait l'hôpital. Il conviendrait par ailleurs de restreindre l'accès aux études de médecine à ceux qui ont pour cela les talents nécessaires, la rapidité de compréhension notamment, et qui se plient à un mode de vie ascétique, rien ne devant venir distraire l'étudiant de ses études. Avant tout, il ne devrait pas être admissible que l'on étudie la médecine seulement pour gagner de l'argent. Si un étudiant impécunieux présente toutes les qualités requises, il devrait pouvoir bénéficier d'un soutien financier. Un système d'examens intermédiaires, assortis de distinctions, aurait l'avantage de stimuler l'émulation entre les étudiants. Enfin - troisième point - les gouvernants devraient soumettre à un examen les médecins qui exercent déjà ${ }^{5}$.

La critique que fait Ibn Ğumay' de l'état de la médecine de son temps était-elle justifiée? Il faut convenir avec Gotthard Strohmaier que l'histoire de la pensée médicale à l'époque médiévale, dans le monde islamique mais aussi dans le monde byzantin, était faite « de progrès et d'apports complémentaires minimes, de synthèses simplificatrices et d'un recours sporadique aux textes originaux de Galien $»^{6}$. L'exigence d'un retour aux sources originelles, formulée par Ibn Ğumay', n'est donc nullement une nouveauté ; le médecin égyptien Ibn Riḍwān, par exemple, l'avait déjà formulée au $\mathrm{XI}^{\mathrm{e}}$ siècle ${ }^{7}$. Quant à la description des agissements contemporains des médecins ignares ainsi que celle des examens qu'il conviendrait de faire subir à ces praticiens, il s'agit pour l'essentiel d'emprunts au De optimo medico cognoscendo de Galien ${ }^{8}$. Les mesures préconisées par Ibn Ğumay pour sélectionner et aider matériellement les étudiants, en revanche, paraissent être originales.

5 La qualité de la formation médicale était très variable ${ }^{9}$. Une multitude de médecins plutôt mal formés exerçaient ainsi au bazar, ouvrant leur cabinet dans une simple échoppe ${ }^{10}$. À Bagdad, par exemple, ce sont plus de 860 médecins qui, selon certaines sources, étaient en activité en 931, parmi lesquels quelques-uns disposaient d'un savoir médical extrêmement réduit ${ }^{11}$. Cependant, une offre de soins étendue et de grande qualité était également disponible, principalement dans les hôpitaux. Ces derniers, aux $\mathrm{XII}^{\mathrm{e}}$ et XIII ${ }^{\mathrm{e}}$ siècles, étaient en général financés par des donations ${ }^{12}$. En Syrie, le centre de l'activité et de la formation médicales était l'hôpital édifié en 1154 à Damas par le prédécesseur de Saladin, Nūr ad-Dīn (1118-1174) et qui portait son nom : l'Hôpital anNū $\overline{1}^{13}$. Dans la biographie d'Abū 'l-Mağd b. Abī 'l-Ḥakam, le premier directeur de cette institution, on trouve une description de son emploi du temps quotidien. Le matin, il se rendait régulièrement au chevet des malades en compagnie de ses assistants et des 
infirmiers. Puis il se rendait à la forteresse pour soigner des personnages de haut rang. Ensuite, de retour à l'hôpital, il s'asseyait « dans la grande galerie des colonnes » et s'y faisait apporter les manuels de la bibliothèque de l'hôpital, également fondée par Nūr ad-Dīn. Une foule de médecins s'assemblaient alors autour de lui, lisaient à haute voix des extraits de ces ouvrages et, trois heures durant, s'entretenaient avec lui de questions médicales ${ }^{14}$.

6 Il y avait aussi des professeurs privés. L'un des plus célèbres d'entre eux était ad-Daḩ wār (mort en 1230), qui administrait sa propre école de médecine à Damas ${ }^{15}$. Le récit de l'un de ses étudiants montre que, contrairement à l'affirmation d'Ibn Ğumay', les œuvres originales de Galien n'étaient nullement tombées dans l'oubli : «J'entrepris d'étudier les œuvres de Galien; le maître connaissait parfaitement tout ce qui lui était exposé sur la base de ces œuvres et des autres livres. Les œuvres de Galien lui plaisaient beaucoup. Lorsqu'il entendait les thèses de Galien sur les maladies, leur traitement et les principes fondamentaux de la médecine, il s'exclamait : "Ça c'est un médecin !" »"

7 On ne sait pas avec certitude dans quelle mesure les médecins étaient soumis à une inspection régulière. Ils relevaient en tout cas de la compétence du muhtasib, l'inspecteur du marché. Celui-ci devait veiller au respect des prescriptions de la loi islamique, la šarī’a ; c'est également à lui qu'incombait la surveillance des marchés, des bazars, de l'activité commerciale et de tous les corps de métier ${ }^{17}$. Le manuel de ašŠayzarī (mort en 1193), qui fut lui-même médecin à Alep, expose les attributions du $m u$ htasib. À propos du contrôle des médecins, il est stipulé qu'ils devaient prêter le serment d'Hippocrate devant le muhtasib et faire la preuve de leur maitrise de certaines connaissances médicales de base. Le muhtasib devait conduire l'inspection en s'appuyant sur l'œuvre de Ḥunayn b. Isḥāq L'Examen médical. Quant à l'œuvre de Galien De optimo medico cognoscendo, abondamment citée par Ibn Ğumay' qui voulait en faire la base de l'examen des praticiens qu'il réclamait, la position de aš-Šayzarī est la suivante : «On ne peut attendre d'aucun médecin qu'il mette en œuvre ce qu'il [Galien] prévoit pour eux dans cet ouvrage. $»^{18}$.

8 De fait, il y a fort à parier que le médecin moyen, dans son modeste cabinet, n'était pas au niveau des exigences élevées d'un Ibn Ğumay'. En revanche, l'élite du corps médical - les médecins attachés au service d'un puissant personnage ou travaillant dans les grands hôpitaux - avait une bonne connaissance du corpus galénique. Or, à quelques exceptions près, nos connaissances sur le corps médical aux alentours de 1200 se limitent précisément à ces médecins d'élite, seuls en effet, du fait de leurs capacités et de leur rang social, à être évoqués dans les chroniques intégrer les collections de vies de savants illustres ${ }^{19}$.

9 Nous voudrions présenter dans les pages qui suivent quelques médecins melkites ${ }^{20}$, juifs ou samaritains de la fin du XII ${ }^{e}$ ou du début du XIII ${ }^{e}$ siècle. Pour peu qu'ils aient vécu dans les contrées sous domination musulmane, les fidèles de ces trois communautés religieuses faisaient partie du groupe des dimmis, des "protégés", c'est-à-dire ces sujets non-musulmans qui, selon le droit islamique, étaient des citoyens de seconde classe, mais bénéficiaient par ailleurs d'une très large autonomie dans la gestion des affaires internes de leur communautée ${ }^{21}$. Pour ces catégories, l'administration civile et la profession de médecin représentaient les principales voies d'ascension sociale. En raison du grand nombre de petits émirats ayyoubides que comptait l'espace syrien au sens large, il existait une forte demande de médecins bien formés ${ }^{22}$. 
10 Il est intéressant de noter que le statut des chrétiens orientaux, des juifs et des samaritains dans les États croisés ne différait guère de celui qui était le leur en terre musulmane. Au moment d'établir leur domination dans la région au début du XII ${ }^{\mathrm{e}}$ siècle, les croisés adoptèrent le système en place de subordination des dimmīs. La seule différence était que la classe dominante était désormais formée des chrétiens latins, tandis que les musulmans étaient réduits au rang de sujets ${ }^{23}$. Les médecins locaux étaient manifestement très appréciés des Francs. Le chroniqueur des États croisés Guillaume de Tyr (mort en 1186) affirme ainsi qu'à son grand regret, les princes croisés, sous l'influence de leurs femmes, donnent la préférence aux médecins juifs, melkites, samaritains ou « sarrasins » sur leurs confrères latins, mettant ainsi leur vie à la merci de gens qui, à en croire le chroniqueur, ne connaissent rien à la médecine ${ }^{24}$. L'anecdote célèbre rapportée par Usāma b. Munqid (1095-1188) offre un tout autre son de cloche. Ce dernier relate de façon vivante l'histoire de deux patients soustraits au médecin local qui les soignaient par un médecin latin; tous deux décédèrent dans les plus brefs délais des suites des thérapies de choc - amputation d'une jambe, trépanation - appliquées par ce dernier ${ }^{25}$. Toutefois, Usāma rapporte également quelques cas de traitements réussis à porter au crédit de l'art médical franc et de ses méthodes, inconnus de lui ${ }^{26}$. D'ailleurs, dans les États croisés pas plus qu'ailleurs, aucun homme, qu'il soit d'origine franque ou orientale, ne pouvait exercer la profession de médecin sans s'être au préalable soumis à un examen conduit par un médecin en exercice ni avoir obtenu ensuite de l'évêque latin du lieu la confirmation de rigueur. On inclinera à penser, avec Lawrence Conrad, que cette loi indique que le savoir des médecins francs et orientaux était à peu près comparable ${ }^{27}$. Dans les États croisés, médecins francs et non-francs cohabitaient, quoiqu'il semble que cette cohabitation entre les deux groupes ait été marquée par une concurrence et une rivalité prononcées $^{28}$.

11 Dans les sociétés islamiques prémodernes, il n'y avait guère que deux débouchés possibles pour qui étudiait les sciences naturelles antiques et la philosophie: l'astrologie ou la médecine. Pour Shelomo Goitein, les médecins étaient de la sorte les "porte-flambeaux de la connaissance profane, des propagateurs professionnels de la philosophie et des sciences", formant en leur qualité de disciples des Grecs et d'héritiers d'une tradition universelle une "fraternité spirituelle qui transcendait les barrières de la religion, de la langue et de l'appartenance ethnique $»^{29}$. Les propos de Goitein méritent certainement d'être nuancés. Le célèbre médecin et professeur Raḍī ad-Dīn ar-Raḥbī (mort en 1233) refusait ainsi de faire profiter les non-musulmans de son enseignement ${ }^{30}$, et à la Madrasa al-Mustanșirìya, fondée en 1234 à Bagdad, seuls les musulmans étaient admis au cursus de médecine ${ }^{31}$. Nous allons pourtant tenter de montrer ici que certains médecins non-musulmans faisaient fi des frontières politiques et religieuses et se déplaçaient apparemment sans aucune entrave entre les États croisés chrétiens et le domaine ayyoubide, exerçant partout leur art sans restriction et mettant à profit divers réseaux transfrontaliers.

Commençons par les médecins melkites. Abū Sulaymān Dāwūd naquit (et grandit sans aucun doute) à Jérusalem, alors sous la domination croisée. Il quitta cependant sa ville natale pour s'installer en Égypte où on le voit exercer, vers le milieu du XII ${ }^{e}$ siècle, la profession d'astrologue mais aussi celle de médecin. Quand le roi Amaury (1163-1174) demanda aux maîtres de l'Égypte de lui fournir un médecin pour soigner son fils, atteint de la lèpre, ces derniers lui recommandèrent Abū Sulaymān, qui 
était alors l'un des rares à savoir correctement composer et administrer la thériaque. Ce dernier revint vivre avec sa famille à Jérusalem. Il s'occupa en personne du fils du roi, puis passa le relais à l'un de ses fils, avant de se retirer lui-même dans un monastère. Il semble que dans sa retraite, Abu Sulaiman renoua avec ses recherches astrologiques et prophétisa la conquête de la ville par Saladin. Il fit parvenir sa prophétie au sultan, qui promit alors de réserver à sa famille un traitement de faveur après la chute de Jérusalem, si ladite prophétie devait s'avérer exacte. Lorsque les faits vinrent donner raison à la prédiction, Saladin invita Abū Sulaymān à sa cour au Caire et lui promit de prendre soin de ses fils, dont quatre étaient également médecins ${ }^{32}$. De fait, trois au moins, dans les décennies qui suivirent, furent attachés au service de membres de la dynastie ayyoubide ${ }^{33}$. C'est donc sans aucune difficulté qu'Abū Sulaymān et ses fils troquèrent un maître musulman pour un maître chrétien et inversement. Dans un contexte où la chute de Jérusalem paraissait inévitable à plus ou moins brève échéance, l'anecdote de la prophétie pourrait également être interprétée comme une tentative d'Abū Sulaiman pour préparer l'avenir : en prenant contact avec Saladin avant même la chute de la ville, il espérait peut-être garantir la position de sa famille.

13 La biographie de Muwaffaq ad-Dīn Ya'qūb b. Siqlāb (mort en 1228), qui exerçait également la médecine à Jérusalem dans les années 1180, nous donne un aperçu des possibilités concrètes qui s'offraient dans le royaume de Jérusalem aux melkites désireux d'étudier les sciences grecques. Ya'qūb $b$. Siqlāb se forma auprès d'un moine qualifié de "philosophe », vivant dans un monastère du désert de Judée. C'est là que Ya'qūb b. Siqlāb fut instruit dans les sciences naturelles, la géométrie, l'arithmétique et l'astrologie. Par la suite, il étudia la médecine à Jérusalem auprès d'un médecin chrétien du nom d'Abū Manșūr. Les sources soulignent en particulier la connaissance qu'avait Yaq'ūb des écrits de Galien, écrits que, affirment-elles, il savait par cœur. La documentation indique qu'il répondait invariablement aux questions médicales de la façon suivante: "À ce propos, Galien disait ceci ou cela". À en croire ces mêmes sources, il connaissait également le grec, traduisait du grec vers l'arabe et aurait même possédé certains ouvrages de Galien en langue originale ${ }^{34}$.

14 Peu après la conquête de Jérusalem par Saladin en 1187, il se rendit à Damas. C'est dans cette ville que le médecin personnel de Saladin, Muwaffaq ad-Dīn b. al-Muțrān (mort en 1191), un personnage dont nous reparlerons, devint son mentor. À son arrivée à Damas, Ya'qūb arborait la tenue typique du médecin franc. Manifestement, il avait exercé à Jérusalem en qualité de médecin officiellement habilité ${ }^{35}$. Lorsque Ibn al-Muțrān le vit ainsi vêtu, il lui représenta que de tels atours n'étaient guère propices à l'avancement de sa carrière ; il lui procura une tenue de médecin damascène et s'entremit pour que lui fût confié le suivi médical de l'émir Fāris ad-Dīn Maymūn al-Qașrīis ${ }^{36}$. De la sorte, Ya'qūb parvint à se faire admettre dans les hautes sphères de la société. Le maître de la Syrie al-Mu'az̧z̧am fit même de Ya'qūb son médecin personnel, et alla jusqu'à lui proposer un poste de gouverneur, qu'il refusa ${ }^{37}$. Un médecin melkite originaire de Jérusalem la franque, mais qui y avait été formé par ses coreligionnaires plutôt que par des Francs, pouvait donc tout à fait s'élever au niveau des médecins issus des célèbres écoles de médecine damascènes et s'introduire dans les plus hautes sphères; il aurait même pu faire une carrière politique sans pour autant avoir à se convertir à l'islam.

15 Pour sa part, Muwaffaq ad-Dīn b. al-Muțrān (mort en 1191), déjà cité, avait embrassé l'islam $^{38}$. La gloire mondaine fut-elle sa seule motivation, comme le prétend BarHebraeus (1228-1288) $)^{39}$ ? On ne le sait. S'il ne s'était pas converti, il lui aurait été 
vraisemblablement plus difficile de s'élever au rang de médecin le plus renommé de son temps et d'acquérir la confiance particulière de Saladin (au point que ce dernier le nomma chambellan (hağib) et tenait à ce qu'il l'accompagnât partout). Selon toute apparence, son père, Abū 'l-Fatḥilyās b. Ğirğis, avait été métropolite (muțrān) melkite de Damas, en même temps qu'un brillant médecin. Pour approfondir ses connaissances, il avait entrepris divers voyages, notamment à Byzance ${ }^{40}$. Les deux frères d'Ibn al-Mut rān exerçaient également comme médecins et restèrent apparemment fidèles à leur foi. Cela dit, ni l'un ni l'autre ne semble avoir fait carrière à la cour d'un personnage de haut rang ${ }^{41}$. Eu égard aux liens étroits qui, en dépit de sa conversion, continuaient à unir Ibn al-Muțrān à la communauté melkite de Damas, ne serait-ce que par sa famille, ce n'est sans doute pas un hasard que le melkite Ya'qūb b. Siqlāb se soit adressé à lui après son installation à Damas. L'Église melkite constituait ainsi un réseau favorisant l'échange des connaissances et la mobilité des personnes par delà les frontières.

16 Les médecins juifs constituent le second groupe qui doit ici retenir notre attention. En Égypte surtout, ces derniers avaient largement pris la relève des médecins chrétiens au $\mathrm{XII}^{\mathrm{e}}$ siècle comme groupe dominant parmi les praticiens non-musulmans ${ }^{42}$. On trouve également trace de quelques médecins juifs dans les États croisés. Ils y furent cependant en butte à l'hostilité, surtout dans les milieux ecclésiastiques. Guillaume de Tyr, comme on l'a vu plus haut, s'en prenait encore de façon indifférenciée à l'ensemble des médecins non-francs, et justifiait son rejet par l'ignorance présumée de ces praticiens; il s'agit là du parti pris d'un savant formé en Europe occidentale et d'un digne représentant de la scolastique française alors en plein développement. Prononcée en 1259 lors d'un concile réuni à Jaffa, l'interdiction faite aux chrétiens de se faire soigner par des juifs ou des « sarrasins » a en revanche une justification religieuse. Les actes conciliaires parlent d'un comportement en contradiction avec le droit canon. Dans la mesure où juifs et «sarrasins » ne font pas appel aux médecins chrétiens - ce par quoi il faut ici entendre, manifestement, les médecins latins - le discrédit serait par là même jeté sur la religion chrétienne, est-il encore précisét ${ }^{43}$. Cette prescription ne semble toutefois pas avoir été suivie d'effets. On ne voit pas que des restrictions aient été imposées aux médecins juifs à la suite du concile ${ }^{44}$.

17 En la personne d'Ibn Ğumay', nous avons déjà présenté l'un des plus importants médecins juifs de cette époque ${ }^{45}$. On ne saurait ici passer sous silence Maïmonide (1138-1204), qui vint en 1165 en Égypte et s'installa à Fusțāt - c'est-à-dire au VieuxCaire. Il déclina l'invitation du roi Amaury à venir à sa cour à Jérusalem, préférant rester en Égypte, qui était alors l'un des principaux centres scientifiques du monde islamique. À compter du début du règne de Saladin en 1171, il fut au service des Ayyoubides en qualité de médecin. Parallèlement, il assuma par deux fois, à de longues années d'intervalle, la charge de chef de la communauté juive d'Égypte (nagid) ${ }^{46}$. Sa célèbre lettre à un savant d'Espagne qui lui avait demandé s'il pouvait devenir son élève donne un aperçu extrêmement vivant du quotidien d'un médecin de cour. Maïmonide répondit que son solliciteur ne saurait nourrir l'espoir de pouvoir s'entretenir avec lui de questions scientifiques ne serait-ce qu'une heure par jour; tous les matins, il devait en effet se rendre de Fusțāt au palais du sultan au Caire, où il lui fallait rester jusqu'à l'après-midi, même lorsqu'aucun cas de maladie ne s'était déclaré. De retour chez lui, il trouvait une salle d'attente bondée, où les juifs côtoyaient les non-juifs, et les petites gens les personnages de haut rang; les consultations l'accaparaient ensuite jusqu'à la nuit tombée. Lorsque le sabbat arrivait et qu'il pouvait délaisser son activité médicale, il avait à s'occuper toute la journée des affaires de sa communauté ${ }^{47}$. Pendant plusieurs 
générations, les descendants de Maïmonide suivirent son exemple, exerçant le métier de médecin tout en présidant aux destinées de la communauté juive ${ }^{48}$. Les médecins non-musulmans appelés à diriger leur communauté religieuse n'étaient pas rares. Outre le père d'Ibn al-Muțān, le métropolite de Damas Abū 'l-Fatḥ Ilyās b. Ğirğis, signalons le patriarche grec-orthodoxe de Jérusalem Jean VIII, qui exerçait comme médecin au début du XII siècle, ou encore, au XIII siècle, le chroniqueur syriaque et maphrien de l'Orient Bar-Hebraeus, qui exerçait la médecine alors qu'il n'était encore qu'évêque $^{49}$. Les fonctions dirigeantes qu'ils venaient à occuper au sein de leur communauté religieuse leur permettaient d'entretenir un réseau de relations géographiquement très étendu.

18 L'histoire de la famille d'Elijah ben Zekarya illustre de façon particulièrement frappante la mobilité des dimmīs au-delà des frontières politiques. Après 1187, la famille quitta tout d'abord l'Égypte pour s'établir à Jérusalem, de nouveau accessible aux juifs ${ }^{50}$. Abū Zirkī, l'un des fils d'Elijah, était également médecin et trouva une place à la cour, sans doute grâce aux relations de son père. Quelque temps plus tard, le père revint au Caire, tandis qu'un autre fils s'installa dans la ville franque d'Acre. Multipliant les voyages entre l'Égypte et la Palestine, les différents membres de la famille se rendaient mutuellement visite et entretenaient par ailleurs une correspondance suivie : les archives de la Geniza nous ont conservé plus de cent de leurs lettres ${ }^{51}$.

19 Le dernier groupe qu'il nous faut évoquer ici est celui des samaritains. Le centre de la vie samaritaine, où résidait le Grand Prêtre et où était célébrée tous les ans la fête de Pâque, était la ville de Naplouse dans le royaume de Jérusalem. Acre et Damas abritaient également d'importantes communautés samaritaines. Vers 1200, quelques samaritains au moins avaient acquis au sein de la société musulmane de Syrie une influence et une aisance matérielle inédites ${ }^{52}$. Deux exemples suffiront ici à l'illustrer. Muhad dِabad-Dīn Yūsuf b. Abī Sa'îd (mort en 1227) dut son ascension sociale à la guérison de la sœur de Saladin. Il fut introduit à la cour du gouverneur de Baalbek alAmğad Bahrāmšah, un petit-neveu de Saladin. Bientôt devenu le principal conseiller du gouverneur, il jouissait de la confiance de ce dernier au point d'être nommé vizir et de se voir confier les rênes du gouvernement. Sa réussite lui valut l'inimitié des musulmans, qui l'accusèrent de malversations et de favoritisme au bénéfice de ses coreligionnaires samaritains et au détriment des musulmans ; ils finirent par obtenir son renvoi et son incarcération ${ }^{53}$. Nous tenons là un très bel exemple de propagande dirigée contre les dimmis, une propagande que l'on voit poindre et resurgir sans cesse, pour des motifs analogues ${ }^{54}$.

20 Mais même la conversion ne prémunissait pas toujours contre la chute. Amīn ad-Daula, le neveu de Muhadِdabad-Dīn, avait suivi son oncle à Baalbek et avait également exercé comme médecin dans la ville. Il s'était rapidement converti à l'islam; son oncle lui aurait alors conseillé d'émigrer dans le territoire sous domination franque s'il venait à l'avenir à regretter sa décision et à vouloir renouer avec son ancienne foi ${ }^{55}$. Amin adDaula gravit l'échelle sociale jusqu'à devenir vizir du maître de Damas aș-Ṣāliḥ Ismā'īl. Mais peu de temps après que ce dernier fut expulsé de Damas, Amin ad-Daula fut jeté en prison puis finalement exécuté en $1251^{56}$. Dans ce cas précis, la religion ne joua certainement aucun rôle; il s'agissait plutôt de rivalités politiques. Les dangers que comportait la position de haut fonctionnaire expliquent sans doute que Yaq'üb $b$. Siqlāb, évoqué plus haut, ait refusé la charge qui lui était proposée. Peut-être le fait 
qu'il n'ait pas été converti à l'islam joua-t-il un rôle en ce sens qu'il y a quelque raison de penser que le risque devait être plus élevé encore pour un non-musulman.

21 Pour les médecins appartenant aux minorités religieuses, le moyen le plus sûr pour faire carrière et, ce faisant, de s'élever dans la hiérarchie sociale à l'époque des croisades était manifestement d'appartenir à une famille de médecins ${ }^{57}$. Avant d'être recommandé pour la prise en charge médicale d'un personnage de haut rang, un jeune homme recevait son enseignement d'un père ou d'un oncle employé à la cour ou introduit dans les cercles dirigeants. En cas de succès, le jeune protégé pouvait espérer à brève échéance un emploi fixe dans l'une ou l'autre $\operatorname{cour}^{58}$. Si le jeune médecin, à l'instar de Yaq'ūb b. Siqlāb, était dépourvu de soutiens familiaux, il était alors contraint de s'appuyer sur des réseaux de relations extrafamiliaux pour trouver un mentor influent.

22 L'exemple des médecins, qui, du simple fait de leur profession, constituent un groupe transfrontalier au sens où l'entend Goitein, a tout particulièrement mis en évidence la complexité du réseau de relations dans lequel évoluaient chrétiens orientaux, juifs et samaritains en Égypte et en Syrie. Pour reprendre un concept proposé par Lutz RichterBernburg, on peut aller jusqu'à dire qu'ils menaient une " existence déchirée » ("split existence »), marquée par une constante, qu'ils vivent sous la domination franque ou musulmane : leur affiliation à une minorité juridiquement subordonnée ${ }^{59}$. Ce statut ne correspondait cependant pas toujours à une limitation de leurs libertés et de leurs marges de manœuvre effectives, et ce serait nier la réalité historique que de confiner ces minorités religieuses dans un rôle de victimes. Le conseil prodigué par Muhadd dab ad-Dīn à son neveu Amīn ad-Daula au sujet de sa conversion évoqué précédemment montre que les membres des minorités religieuses savaient parfaitement exploiter la situation politique à leur avantage ${ }^{60}$. La conversion pouvait également fonctionner dans l'autre sens. C'est ce qu'il ressort d'une clause des Assises du Royaume de Jérusalem (début du XII ${ }^{\mathrm{e}}$ siècle), aux termes de laquelle les enfants étaient en droit de déshériter ${ }^{61}$ leurs parents convertis par le passé au christianisme si ces derniers avaient ensuite émigré en terre musulmane pour renouer avec leur foi juive, "sarrasine ", voire, selon la variante de l'un des manuscrits, samaritaine ${ }^{62}$. En fonction des nécessités du moment, les membres des minorités religieuses pouvaient donc virer de bord. Les liens familiaux et la cohésion interne des différents groupes religieux créaient, par delà les frontières, un réseau susceptible de faire office de filet de sécurité. Les personnalités placées à la tête de ces communautés, souvent elles-mêmes médecins de profession comme on l'a vu, contribuaient à la constitution de tels réseaux par leurs relations personnelles, qui s'étendaient bien au-delà du cadre régional. On en veut pour preuve l'exemple de Maïmonide, vers qui convergeaient d'innombrables requêtes en tout genre en provenance de l'ensemble du monde juif;c'est aussi ce que montrent, indirectement, les bons offices d'Ibn al-Muțrān en faveur de son obligé Yaq'ub. Par là même, les minorités religieuses constituaient un pont entre les États croisés et l'empire ayyoubide, contribuant ainsi à renforcer la cohérence de l'espace syro-égyptien au temps des croisades - notamment dans le domaine de l'érudition médicale ${ }^{63}$. 


\section{BIBLIOGRAPHIE}

\section{Sources}

Abū Šāma, Tarāğim riğāl al-qarnayn as-sādis wa-s-sābi' al-ma'rūf bi-ḍ-ḍyl 'alā arrauḍatayn, éd. par Muḥammad Kauțarī, Le Caire 1947.

Alī b. al-'Abbās al-Mağūsī, Kāmil aș-șinā'a aț-țibbīya, Bulāq 1294H./1877.

Alī b. al-'Abbās al-Mağūsī, Kāmil aș-șinā‘a aț-țibbīya al-ma'rūf bi-l-kitāb al-malakī, éd. par Fuat Sezgin (fac-similé de l'édition de 1877), Francfort 1985 (Publications of the Institute for the History of Arabic-Islamic Science, series C, Facsimile Editions, 16).

aš-Šayzari, Nihāyat ar-rutba fi țalab al-ḥisba, éd. par al-Bāz al-'Arīnī, Le Caire 1946 ; trad. angl. : The Book of the Islamic Market Inspector, trad. par R. P. Buckley, Oxford 1999 (Journal of Semitic Studies, Supplement, 9).

Assises de la Cour des Bourgeois dans : Recueil des Historiens des Croisades, Lois, t. 2, éd. par A. Beugnot, Paris 1843.

Galenus, De optimo medico cognoscendo (On examinations by which the best physicians are recognized), éd. et trad. par A. Z. Iskandar, Berlin 1988 (Corpus medicorum Graecorum. Supplementum orientale, 4).

Gregory Abu'l Faraj, The Chronography of Gregory Abu'l Faraj, the Son of Aaron, the Hebrew Physician Commonly Known as Bar Hebraeus, Being the First Part of his Political History of the World, éd. et trad. par E. A. Wallis Budge, t. 1, London 1932 (réimpr. Amsterdam 1976).

Guillaume de Tyr, Chronicon, éd. par R. B. C. Huygens, t. 2, Turnhout 1986 (Corpus Christianorum. Continuatio mediaevalis, 63A).

Ibn Abī UȘaybi‘a, Kitāb 'uyūn al-anbā' fi țabaqāt al-ațibba', éd. par A. Müller, t. 1, Le Caire 1882 ; t. 2, Königsberg 1884.

Ibn Abī UȘaybi'a, Kitāb 'uyūn al-anbā' fi țabaqāt al-ațibba', éd. par Nizār Riḍā, Beyrouth 1965.

Ibn al-Qifțī, Ta'rīh al-ḥukamā', éd. par J. Lippert, Leipzig 1903.

Ibn Ğumai', al-Maqāla aș-șalāhīya fi ihyā’ aș-șinā'a aț-țibbīya, éd. et trad. par H. Fähndrich, Wiesbaden 1983 (Abhandlungen für die Kunde des Morgenlandes, 46.3).

Ibn Wāṣil, Mufarriğ al-kurūb fi ahbbār Banī Ayyūb, t. 5, éd. par Ḥasanain M. Rabī‘ et Sa'īd A. 'Āšūr, Le Caire 1977.

Usāma b. Munqị, Kitāb al-i'tibār, éd. par Ph. K. Hitti, Princeton 1930; trad. angl. : Memoirs of an Arab-Syrian Gentleman, trad. par Ph. K. Hitti, New York 1927; trad. all. : Ein Leben im Kampf gegen Kreuzritterheere, trad. par G. Rotter, Bâle 1978.

\section{Littérature secondaire}

Bosworth, C. E. (1979-1980) : "The "Protected Peoples" (Christians and Jews) in Medieval Egypt and Syria ", Bulletin of the John Rylands University Library, 62, p. 11-36 (repr. dans : Id. : The Arabs, Byzantium and Iran. Studies in Early Islamic History and Culture, London, 1996 [= Collected Studies Series, CS 529]). 
Cahen, C. (1934) : «Indigènes et Croisés. Quelques mots à propos d'un médecin d'Amaury et de Saladin », Syria, 15, p. 351-360.

Cheikho, L. (1983) : 'Ulamā' an-nașrānīya fi 'I-islām (Les savants arabes chrétiens en Islam), 622-1300, éd. revue par C. Hechaïme, Jounieh-Rome (Patrimoine arabe chrétien, 5).

Cheikho, L. (1987) : Wuzarā' an-nașrānīya wa-kuttābuhā fí 'I-islām (Les vizirs et secrétaires arabes chrétiens en Islam), 622-1517, éd. revue par C. Hechaïme, Jounieh (Patrimoine arabe chrétien, 11).

Cohen, M. R. (1989) : « Maimonides' Egypt », dans : Ornsby, E. L. (éd.) : Moses Maimonides and His Time, Washington (Studies in Philosophy and the History of Philosophy, 19), p. 21-34.

Cohen, M. R. (1993) : « The Burdensome Life of a Jewish Physician and Communal Leader: A Geniza Fragment from the Alliance Israélite Universelle Collection », Jerusalem Studies in Arabic and Islam, 16, p. 125-136.

Conrad, L. I. (1995) : « The Arabic Medical Tradition », dans : Conrad, L. I. / Neve, M. / Nutton, V. / Porter, R. / Wear, A. (éd.) : The Western Medical Tradition 800 BC to AD 1800, Cambridge, p. 93-138.

Conrad, L. I. (1999) : « Usama ibn Munqidh and Other Witnesses to Frankish and Islamic Medicine in the Era of the Crusades ", dans : Amar, Z. / Lev, E. / Schwartz, J. (éd.) : Medicine in Jerusalem throughout the Ages, Tel Aviv, p. XXVII-LII.

Dolev, E. (1996) : « Medicine in the Crusaders' Kingdom of Jerusalem », dans : Waserman, M. / Kottek, S. S. (éd.) : Health and Disease in the Holy Land. Studies in the History and Sociology of Medicine from Ancient Times to the Present, Lewiston-Queenston-Lampeter, p. 157-172.

Drory, J. (1991) : « The Early Decades of Ayyūbid Rule », dans : Kraemer, J. L. (éd.) : Perspectives on Maimonides. Philosophical and Historical Studies, Oxford (Littman Library of Jewish Civilization), p. 293-302.

Elisséeff, N. (1967) : Nūr ad-Dīn. Un grand prince musulman de Syrie au temps des croisades (511-569 H.) 111861174), t. 3, Damas.

Fattal, A. (1958) : Le Statut légal des non-musulmans en pays d'Islam, Beyrouth.

Fiey, J. M. (1993) : Pour un Oriens Christianus Novus. Répertoire des diocèses syriaques orientaux et occidentaux, Beyrouth (Beiruter Texte und Studien, 49).

Goitein, S. (1971) : A Mediterranean Society. The Jewish Communities of the Arab World as Portrayed in the Documents of the Cairo Geniza, t. 2 : The Community, Berkeley / Los Angeles / London.

Hamarneh, S. K. (1964) : « Origins and Functions of the Hisba System in Islam and its Impact on the Health Professions ", Sudhoffs Archiv, 48, p. 157-173 (repr. dans : HealthSciences in Early Islam. Collected Papers by Sami K. Hamarneh, t. 1, éd. par A. Anees, Blanco, 1983, p. 113-125).

Hamarneh, S. K. (1966) : « Muslim Historiography as Related to the Health Professions », Sudhoffs Archiv, 50, p. 2-24 (repr. dans : HealthSciences in Early Islam. Collected Papers by Sami K. Hamarneh, t. 1, éd. par A. Anees, Blanco, 1983, p. 229-246).

Harvey, S. (1991) : « Maimonides in the Sultan's Palace », dans : Kraemer, J. L. (éd.) : Perspectives on Maimonides. Philosophical and Historical Studies, Oxford (Littman Library of Jewish Civilization), p. 47-75.

Hau, F. R. (1978) : « Die Bildung des Arztes im islamischen Mittelalter », Clio Medica, 13, p. 95-124 et $175-200$.

Humphreys, R. S. (1977) : From Saladin to the Mongols. The Ayyubids of Damascus, 1193-1260, Albany. 
Issa, A. (1928) : Histoire des Bimâristans (hôpitaux) à l'époque islamique, Le Caire.

Jacquart, D. / Micheau, F. (1990) : La Médecine arabe et l'occident médiéval, Paris (Collection IslamOccident, 7).

Jadon, S. (1970a) : « The Physicians of Syria during the Reign of Salah al-Dīn 570-589 A.H. 1174-1193 A.D. ", Journal of the History of Medicine, 25, p. 323-340.

Jadon, S. (1970b) : « A Comparison of the Wealth, Prestige and Medical Works of the Physicians of Salah al-Dīn in Egypt and Syria », Bulletin of the History of Medicine, 44, p. 64-75.

Kedar, B. Z. (1989) : « The Frankish Period », dans : Crown, A. D. (éd.) : The Samaritans, Tübingen, p. 82-94.

Klein-Franke, F. (1982) : Vorlesungen über die Medizin im Islam, Wiesbaden (Sudhoffs Archiv. Beihefte, 23).

Klein-Franke, F. (1996) : "Health and Disease in Medieval Muslim Palestine », dans : Waserman, M. / Kottek, S. S. (éd.) : Health and Disease in the Holy Land. Studies in the History and Sociology of Medicine from Ancient Times to the Present, Lewiston / Queenston / Lampeter, p. 103-134.

Kohlberg, E. / Kedar, B. Z. (1988) : « A Melkite Physician in Jerusalem and Ayyubid Damascus: Muwaffaq ad-Dīn Ya'qūb ibn Siqlāb», dans : Kedar, B. Z. / Udovitch, A. L. (éd.) : The Medieval Levant. Studies in Memory of Eliyahu Ashtor(1914-1984), s. 1.(Asian and African Studies, 22).

Leclerc, L. (1876) : Histoire de la médecine arabe, t. 2, Paris (réimpr. New York, 1960).

Leiser, G. (1983) : « Medical Education in Islamic Lands from the Seventh to the Fourteenth Century ", Journal of the History of Medicine, 38, p. 48-75.

Meyerhof, M. (1929) : «L'œuvre médicale de Maimonide », dans : Archeion, 11, p. 136-155 (repr. dans : Id. : Studies in Medieval Arabic Medicine, London, 1984 [Collected Studies Series, CS 204]).

Meyerhof, M. (1938) : « Medieval Jewish Physicians in the Near East, from Arabic Sources », Isis, 28, p. 432-460 (repr. dans : Id. : Studies in Medieval Arabic Medicine, London, 1984 [Collected Studies Series, CS 204]).

Meyerhof, M. (1945) : «Sultan Saladin's Physician on the Transmission of Greek Medicine to the Arabs ", Bulletin of the History of Medicine, 18, p. 169-178 (repr. dans : Id. : Studies in Medieval Arabic Medicine, London, 1984 [Collected Studies Series, CS 204]).

Motzkin, A. L. (1965) : The Arabic Correspondence of Judge Elijah and His Family. A Chapter in the Social History of Thirteenth Century Egypt, PhD University of Pennsylvania.

Motzkin, A. L. (1970) : «A Thirteenth-Century Jewish Physician in Jerusalem (A Geniza Portrait) », Muslim World, 60, p. 344-349.

Nasrallah, J. (1974) : « Médecins melchites de l'époque Ayyubide », Parole de l'orient, 5, p. 189-200.

Nasrallah, J. (1983) : Histoire du mouvement littéraire dans l'église melchite du Ve au XXe siècle.

Contribution à l'étude de la littérature arabe chrétienne, t. 3/1 (969-1250), Louvain / Paris.

Pahlitzsch, J. (2001) : Graeci und Suriani im Palästina der Kreuzfahrerzeit. Beiträge und Quellen zur Geschichte des griechisch-orthodoxen Patriarchats von Jerusalem, Berlin (Berliner Historische Studien 33, Ordensstudien 15).

Pahlitzsch, J. / Weltecke, D. (2001) : « Konflikte zwischen den nicht-lateinischen Kirchen im Königreich Jerusalem », dans : Bauer, D. / Herbers, K. / Jaspert, N. (éd.) : Jerusalem im Hoch- und Spätmittelalter. Konflikte und Konfliktbewältigung - Vorstellungen und Vergegenwärtigungen, Francfort / New York (Campus Historische Studien, 29), p. 119-145. 
Pahlitzsch, J. (2004) : «The Transformation of Latin Religious Institutions into Endowments by Saladin in Jerusalem ", dans : Korn, L. / Pahlitzsch, J. (éd.) : Governing the Holy City. The Interaction of Social Groups in Jerusalem between the Fatimid and Ottoman Period, Wiesbaden.

Prawer, J. (1980) : Crusader Institutions, Oxford.

Prawer, J. (1988) : The History of the Jews in the Latin Kingdom of Jerusalem, Oxford.

Richter-Bernburg, L. (1996) : «St. John of Acre - Nablus - Damascus : The Samaritan Minority under Crusaders and Muslims ", dans : Beltz, W. (éd.) : Die Folgen der Kreuzzüge für die orientalischen Religionsgemeinschaften. Internationales Kolloquium vom 16-18.10.1996 in Halle/Saale, Halle (Hallesche Beiträge zur Orientwissenschaft, 22), p. 117-130.

Rosenthal, F. (1978) : "The Physician in Medieval Muslim Society », Bulletin of the History of Medicine, 52, p. 475-491 (repr. dans : Id. : Science and Medicine in Medieval Islam. A Collection of Essays, Aldershot / Brookfield, 1990 [Collected Studies Series, CS 330]).

Rosner, F. (1998) : The Medical Legacy of Moses Maimonides, Hoboken.

Savage-Smith, E. (1996) : « Medicine », dans : Rashed, R. / Morelon, R. (éd.) : Encyclopedia of the History of Arabic Science, t. 3, London / New York, p. 903-962.

Schacht, J. / Meyerhof, M. (1937) : The Medico-Philosophical Controversy Between Ibn Butlan of Baghdad and Ibn Ridwan of Cairo. A Contribution to the History of Greek Learning Among the Arabs, Le Caire (The Egyptian University, The Faculty of Arts, Publication, 13).

Sivan, E. (1967) : « Note sur la situation des chrétiens à l'époque ayyubide », Revue de l'histoire des religions, 86, p. 117-130.

Spies, O. / Müller-Bütow, H. (1971) : Anatomie und Chirurgie des Schädels, insbesondere der Hals-, Nasen- und Ohrenkrankheiten nach Ibn al-Quff, Berlin / New York (Ars Medica, III. Abt. 1).

Stitskin, L. D. (1977) : Letters of Maimonides, New York.

Strohmaier, G. (1987) : « Rezension zu Hartmut Fähndrichs Edition von Ibn Gumai’s al-Maqala assalahiya », Orientalistische Literaturzeitung, 82, p. 374-375.

Strohmaier, G. (1996a) : «Die Rezeption und die Vermittlung : die Medizin in der byzantinischen und in der arabischen Welt », dans : Grmek, M. D. (éd.) : Die Geschichte des medizinischen Denkens. Antike und Mittelalter, München, p. 151-181.

Strohmaier, G. (1996b) : " „Von Alexandria nach Bagdad“ - eine fiktive Schultradition », dans : Id. : Von Demokrit bis Dante. Die Bewahrung antiken Erbes in der arabischen Kultur, Hildesheim / Zurich /New York, p. 313-322 (publié en premier lieu dans : Wiesner, J. [éd.] : Aristoteles - Werk und Wirkung. Paul Moraux gewidmet, t. 2, Berlin / New York, 1987, p. 380-389).

Strohmaier, G. (1996c) : « Ärztliche Ausbildung im islamischen Mittelalter », dans : Id. : Von Demokrit bis Dante. Die Bewahrung antiken Erbes in der arabischen Kultur, Hildesheim / Zurich / New York, p. 391-396 (publié en premier lieu dans : Klio, 61, 1979, p. 519-524).

Terzioglu, A. (1976) : « Das Nureddin-Krankenhaus in Damaskus (gegr. 1154) aus der Epoche der Seldschuken und seine Bedeutung für die Medizin- und Krankenhausgeschichte », Historia Hospitalium, 11, p. 59-75.

Ullmann, M. (1970) : Die Medizin im Islam, Leiden / Köln (Handbuch der Orientalistik 1. Abt. : Der Nahe und Mittlere Osten, Ergänzungsbd. 6, 1. Abschnitt).

Ullmann, M. (1978) : Islamic Medicine, Edimbourg (Islamic Surveys, 11). 
Wedel, G. (2000) : « Transfer of Knowledge and the Biographies of Samaritan Scholars : Careers of Samaritan Physicians under Muslim Patronage », dans : Morabito, V. / Crown, A. D. / Davey, L. (éd.) : Samaritan Researches. Proceedings of the Congress of the SES (Milan, July 8-12 1996) and of the Special Section of the ICANAS Congress (Budapest, July 7-11 1997), Sydney, t. 5, p. 3.7-3.83.

Wedel, G. (2001) : «Medicine as an Integrative Factor in Medieval Islamic Societies. Physicians as Mediators in Multi-Religious, Multi-Ethnical and Multi-Social Environments ? ", dans : Science and Cultural Diversity. Filling a Gap in the History of Science. Proceedings of the 21th International Congress of History of Science, Mexico City 8-14 July 2001 (inédit).

Wickersheimer, E. (1951) : «Organisation et législation sanitaires au Royaume franc de Jérusalem (1099-1291) », Archives internationales d'histoire des sciences, nouvelle série, 30, p. 687-705.

Woodings, A. F. (1971) : « The Medical Resources and Practice of the Crusader States in Syria and Palestina 1096-1193 », Medical History, 15, p. 268-277.

\section{NOTES}

1. Ibn Ğumai', al-Maqāla aș-șalāḥīya fi ihyā’ aș-șinā‘a aț-țibbīya, éd. Fähndrich, § 2. Sur cet auteur, voir aussi Ullmann (1970), p. 164-165. La toute première étude du tableau de l'histoire de la médicine brossé par Ibn Ğumay‘ est celle de Meyerhof (1945).

2. Ullmann (1978), p. 23-24 ; Klein-Franke (1982), p. 50-51 ; Jacquart / Micheau (1990), p. 55-85 ; Conrad (1995), p. 110-122 ; Savage-Smith (1996), p. 913-927 ; Strohmaier (1996a). Sur le transfert du savoir médical antique d'Alexandrie à Bagdad, voir Strohmaier (1996b).

3. Connu également sous le nom de Kitāb al-malakī: Alī b. al-'Abbās al-Mağūsī, Kāmil aṣșinā'a aț-țibbīya (1877). Fuat Sezgin en a donné un fac-similé : Kāmil aș-șinā'a aț-țibbīya al-ma'rūf bi-l-kitāb al-malakì (1985).

4. Ibn Ğumai', al-Maqāla aș-șalāhịya, éd. Fähndrich, §§ 67-86.

5. Ibn Ğumai', al-Maqāla aș-șalāhīya, éd. Fähndrich, §§ 118-123.

6. Strohmaier (1996a), p. 153.

7. Schacht / Meyerhof (1937), p. 20-29; Rosenthal (1978), p. 484.

8. Seule la traduction arabe a été conservée : Galenus, De optimo medico cognoscendo, éd. Iskandar. Strohmaier (1987), p. 374 sq.

9. Pour un tour d'horizon de la formation des médecins, voir Hau (1978) ; Strohmaier (1996c) ; Leiser (1983).

10. Voir par exemple Goitein (1971), p. 253 ; Jadon (1970a), p. 337 ; Conrad (1995), p. 132. En toute logique, le contrôle de l'activité des médecins était du ressort du muhtasib, «l'inspecteur du marché "; voir à ce propos la note 17. Le revenu du médecin moyen correspondait sans doute à celui du propriétaire d'une échoppe : voir Rosenthal (1978), p. 484.

11. L'inspection des médecins à Bagdad en 931 est évoquée dans Ibn al-Qifțī, Ta'rīh alḥukamā', éd. Lippert, p. 191 sq. et dans Ibn Abī Ușaybi'a, Kitāb 'uyūn al-anbā' fi țabaqāt al-ațibbā', éd. Müller, t. 1, p. 222 (éd. Riḍā, p. 302).

12. Sur la fondation d'hôpitaux par Saladin, voir Pahlitzsch (2004).

13. Issa (1928), p. 97-107 ; Elisséeff (1967), p. 838-843; Terzioglu (1976).

14. Ibn Abī UȘaybi'a, Kitāb 'uyūn al-anbā', éd. Müller, t. 2, p. 155 (éd. Riợā, p. 628). On trouve une traduction allemande de ce passage dans Spies / Müller-Bütow (1971), p. 13 sq.

15. Sur ce personnage, voir Ullmann (1970), p. 172 ; Hau (1978), p. 100.

16. Ibn Abī UȘaybi‘a, Kitāb 'uyūn al-anbā', éd. Müller, t. 2, p. 242 (éd. Riộā, p. 731). Nous suivons ici la traduction allemande dans Spies / Müller-Bütow (1971), p. 14.

17. Hamarneh (1964), p. 167-173. 
18. aš-Šayzarī, Nihāyat ar-rutba fi țalab al-hisba, éd. al-Bāz al-'Arīnī, p. 99 sq. ; trad. angl. (Buckley), p. 114-116: «no physician can be expected to perform what he [Galien] stipulated for them in it ".

19. La principale source pour l'histoire de la médecine en Syrie et en Égypte aux XII ${ }^{\mathrm{e}}$ et XIII ${ }^{\mathrm{e}}$ siècles est la collection de biographies de médecins due à Ibn Abī Ușaybi'a (1203-1270). Sur le genre littéraire des Vies de médecins, voir Hamarneh (1966) ; Conrad (1995), p. 124 ; Wedel (2000), p. 3.76-3.78.

20. Par melkites, on entend généralement les fidèles de l'Église impériale byzantine; voir Pahlitzsch (2001), p. 41.

21. Sivan (1967) ; Bosworth (1979-1980). De façon plus générale sur ce thème, voir Fattal (1958).

22. Goitein (1971), p. 242 ; Richter-Bernburg (1996), p. 118 et 121.

23. Pahlitzsch (2001), p. 181-188.

24. Guillaume de Tyr, Chronicon, éd. Huygens, lib. 18 c. 34, p. 859. D'après Conrad (1999), p. XLIVXLVI, il est impossible de conclure de ce passage que les croisés accordaient généralement la préférence aux médecins orientaux. Selon lui, ces femmes de croisés auraient également été d'origine orientale, ce qui aurait expliqué leur prédilection pour des médecins orientaux qu'elles connaissaient bien.

25. Usāma b. Munqị̂, Kitāb al-i'tibār, éd. Hitti, p. 132 sq. ; trad. angl. (Hitti), p. 162 ; trad. all. (Rotter), p. $151 \mathrm{sq.}$

26. Usāma b. Munqiḍ, Kitāa al-i'tibār, p. 133 sq.; trad. angl. (Hitti), p. 162 sq. ; trad. all. (Rotter), p. 152 sq. Sur la vision qu'a Usāma des médecins francs, voir l'étude approfondie de Conrad (1999), p. XXVII-LII, qui cherche à prouver que l'art médical franc n'était pas très inférieur à la pratique orientale, comme l'affirment les travaux anciens: Wickersheimer (1951); Woodings (1971) ; Dolev (1996).

27. Assises de la Cour des Bourgeois, éd. Beugnot, c. 238, p. 169 ; Conrad (1999), p. L-LI ; Wickersheimer (1951), p. 695 sq. ; Prawer (1988), p. 108, doute toutefois que cette loi, promulguée au milieu du XIIe siècle, ait jamais été appliquée.

28. Dolev (1996), p. 166 ; Conrad (1999), p. XLVI-XLVII, cite en outre l'exemple de Gilbertus Angelicus (Gilbert l'Anglais) ; ce dernier, qui accompagna Richard Cœur de Lion en Terre sainte au début des années 1190, à l'époque de la troisième croisade, rapporte qu'il y guérit un enfant malade après que les «célèbres » médecins musulmans et melkites eurent échoué. Sur la vive concurrence entre médecins en Égypte, voir Goitein (1971), p. 241.

29. Goitein (1971), p. 241.

30. Ibn Abī UȘaybi‘a, Kitāb 'uyūn al-anbā’, éd. Müller, t. 2, p. 193 (éd. Riộā, p. 673).

31. Strohmaier (1996c), p. 396.

32. Ibn Abī UȘaybi'a, Kitāb 'uyūn al-anbā', éd. Müller, t. 2, p. 121 sq. (éd. Riọā, p. 587-589). Cahen (1934); Kohlberg / Kedar (1988), p. 114 sq.; Klein-Franke (1996), p.119-121. Nasrallah (1974) offre également un rapide tour d'horizon des médecins melkites.

33. Ibn Abī UȘaybi'a, Kitāb 'uyūn al-anbā', éd. Müller, t. 2, p. 122 sq. (éd. Riḍā, p. 589 sq.).

34. Ibn Abī UȘaybi'a, Kitāb 'uyūn al-anbā', éd. Müller, t. 2, p. 214-216 (éd. Riọā, p. 697-699). Voir aussi la biographie, plus succincte et en partie divergente, donnée par Ibn al-Qifțī, Ta'rīh al-hukamā', éd. Lippert, p. 378 sq. On trouve une traduction des deux biographies dans Kohlberg / Kedar (1988), p. 121-124. Parmi les livres de Galien en possession de Ya'qūb b. Siqlāb, l' Ars magna et le De morborum causis et symptomatibus libri sex sont nommément cités.

35. Kohlberg / Kedar (1988), p. 119 voient aussi dans cette tenue franque l'habit traditionnel porté par les médecins habilités dans les États croisés. Conrad (1999), p. XLVII-XLVIII suppose en revanche que Ya'qūb entreprit de porter des vêtements francs de sa propre initiative, pour se rapprocher de l'élite franque et élargir ainsi sa clientèle.

36. Ibn Abī UȘaybi‘a, Kitāb 'uyūn al-anbā', éd. Müller, t. 2, p. 177 (éd. Rị̣ā, p. 654). 
37. Ibn Abī UȘaybi'a, Kitāb 'uyūn al-anbā', éd. Müller, t. 2, p. 215 (éd. Riḍā, p. 698 sq.) ; Kohlberg / Kedar (1988), p. 115-120 ; Klein-Franke (1996), p. 123-125 ; Pahlitzsch (2001), p. 209.

38. Ibn Abī UȘaybi'a, Kitāb 'uyūn al-anbā', éd. Müller, t. 2, p. 175-181 (éd. Riḍā, p. 651-659). Leclerc (1876), p. 43-45 ; Ullmann (1970), p. 165 sq. ; Jadon (1970a), p. 330-332 ; Jadon (1970b), p. 68 sq. ; Cheikho (1983), p. 84 sq.

39. Gregory Abu'l Faraj, The Chronography of Gregory Abu'l Faraj, éd. Wallis Budge, p. 329.

40. Ibn Abī UȘaybi'a, Kitāb 'uyūn al-anbā', éd. Müller, t. 2, p. 175 (éd. Riḍā, p. 652). Ibn Abi UȘ aybi'a ne précise pas à quelle Église chrétienne appartient la famille d'Ibn al-Muțrān. Plusieurs éléments penchent en faveur de l'appartenance à l'Église melkite, à commencer par le séjour du père à Byzance - les melkites étant affiliés à l'Église impériale byzantine. Par ailleurs, la liste des évêques syriaques-orthodoxes (jacobites) de Damas attestés pour le XII ${ }^{\mathrm{e}}$ siècle, établie par Fiey (1993), p. 183 («Denys [1129/1137]; Jean à Jérusalem [1168] ; Jean Grégoire [après 1168-avant 1194/1195] »), ne comprend aucun Ilyās/Elias. Nasrallah (1983) range également Ibn al-Muțrān au nombre des melkites, sans donner aucun élément à l'appui de cette affiliation. Enfin, Hibat Allāh b. Yūnis b. Abī 'l-Fatḥ, qui était manifestement un neveu d'Ibn al-Muțrān, appartenait de façon certaine à l'Église melkite : voir infra note 54.

41. Cela aurait vraisemblablement été mentionné par Ibn Abī Ușaybi'a, Kitāb 'uyūn al-anbā', éd. Müller, t. 2, p. 179 sq. (éd. Riḍā, p. 656).

42. Sur les sept médecins de Saladin en Égypte, deux étaient musulmans et les autres juifs : Jadon (1970a), p. 65 ; Meyerhof (1938), p. 443 sq.

43. Mansi (1784), col. 328 sq.

44. Prawer (1988), p. 108 sq.

45. Voir supra note 1.

46. Ullmann (1970), p. 167-170 ; Goitein (1971), p. 244 ; Drory (1991), p. 302 ; Cohen (1989). Sur l'œuvre médicale de Maïmonide, voir Meyerhof (1929) ; Rosner (1998).

47. Stitskin (1977), p. 133-135. Sur la description que fait Maïmonide de son quotidien accablé de travail et l'idéal de vie retirée qu'il lui oppose, voir Harvey (1991).

48. Goitein (1971), p. 244 sq. ; Cohen (1989), p. 25-27; Cohen (1993).

49. Sur cette facette de la vie de Jean VIII, qui était d'origine grecque, et de Bar-Hebraeus, voir Pahlitzsch (2001), p. 122. Un traité rédigé en grec par Jean VIII lui-même et dirigé contre le pain azyme utilisé par les Latins pour l'eucharistie, nous renseigne sur son activité médicale. Dans cet ouvrage, il raconte dans quelles circonstances il en vint à s'entretenir avec un "philosophe » latin - une conversation qui fut selon ses dires à l'origine de son traité. Il rapporte qu'un Latin qui se piquait de philosophie vint le trouver, manifestement accompagné de gardes du corps, pour le prier de lui indiquer un traitement pour sa hanche douloureuse. De son propre aveu, Jean le conseilla du mieux qu'il put et lui administra un certain médicament, sur lequel il ne donne pas plus de précisions. Peu de temps après, l'homme revint voir le médecin pour le remercier. Il ajouta à cette occasion que cela serait merveilleux si Jean pouvait également le guérir de son mal « spirituel »; à l'en croire, il en était venu à douter du bien-fondé de l'utilisation de pains azymes. S'ensuit alors une dispute théologique approfondie sur cette matière, au cours de laquelle Jean réfute naturellement les thèses du Latin, qui, bien entendu, ne se montre guère convaincu. On trouvera une analyse détaillée de ce traité dans Pahlitzsch (2001), p. 120-131.

50. Sur le retour des juifs dans la ville de Jérusalem passée sous contrôle ayyoubide, voir Prawer (1988), p. 64-92.

51. Motzkin (1965); Motzkin (1970); Prawer (1988), p. 81-84.

52. Kedar (1989) ; Richter-Bernburg (1996), p. 119-123.

53. Ibn Abī Ușaybi'a, Kitāb 'uyūn al-anbā', éd. Müller, t. 2, p. 233 sq. (éd. Riọā, p. 721-723). Richter-Bernburg (1996), p. 126 sq., défend la thèse selon laquelle l'accès aux plus hautes charges passait obligatoirement par une conversion préalable à l'islam et, fort de cette affirmation, soutient sans autre preuve que Muhad $\underline{d}$ ab ad-Dīn devait s'être converti. Wedel (Wedel [2000], 
p. 3.79-3.80; Wedel [2001]) suppose que les plus hautes charges restaient fermées aux nonmusulmans pour la simple raison qu'ils ne pouvaient pas commander à des musulmans; mais aussi mineur que fût l'émirat de Baalbek, le vizir y avait bel et bien le pouvoir de donner des ordres aux musulmans. Selon le même auteur, il en serait allé de même dans le cas de Ya'qūb b. Siqlāb si ce dernier avait accepté le poste de gouverneur qu'on lui proposait. Sur les vizirs chrétiens, voir également Cheikho (1987).

54. Hibat Allāh b. Yūnis b. Abī 'l-Fatḥ, qui officiait comme trésorier au service des Ayyoubides, en butte à des griefs similaires - on l'accusa d'avoir renforcé sa position au détriment des musulmans, d'avoir favorisé les chrétiens, d'avoir fait agrandir le cathédrale Notre-Dame de Damas, d'avoir opprimé les musulmans - fut privé de sa charge et enchaîné au portail de l'église Notre-Dame : Abū Šāma, Tarāğim riğāl al-qarnayn as-sādis wa-s-sābi' al-ma'rūf bi-d̆dayl 'alā ar-rauḍatayn, éd. Kautarīī p. 156 ; Nasrallah (1983), p. 345. On pourrait être tenté de faire de ce membre en vue de la communauté melkite de Damas un petit-fils du métropolite Abū 'l-Fatḥ Ilyās b. Ğirğis, le père d'Ibn al-Muțrān, évoqué ci-dessus. On se reportera aux titres cités supra dans la note 21 .

55. Cette anecdote se trouve uniquement dans Ibn Wāșil, Mufarriğ al-kurūb fi aḩbār Banì Ayyūb, éd. Rabī' / 'Āšūr, p. 236 sq. ; Richter-Bernburg (1996), p. 126.

56. Ibn Abī Ușaybi'a, Kitāb 'uyūn al-anbā', éd. Müller, t. 2, p. 234-239 (éd. Riọaā, p. 723-728). Sur sa carrière politique, voir Humphreys (1977), p. 273-280, 289 et 319-320 ; Richter-Bernburg (1996), p. 127 sq. ; Wedel (2000), p. 3.80-3.81.

57. D'autres exemples, à des époques plus anciennes, dans Leiser (1983), p. 49 sq.

58. D'autres exemples dans Goitein (1971), p. 247 sq.

59. Richter-Bernburg (1996), p. 119.

60. Voir également, sur ce sujet, Pahlitzsch / Weltecke (2001).

61. sic (enterben) dans le texte original ( $\mathrm{NdT})$.

62. Prawer (1980), p. 449 (édition du texte), p. 439-457 (analyse de l'ensemble du corpus juridique) ; Prawer (1988), p. 106 ; Richter-Bernburg (1996), p. 119 sq.

63. On ne peut affirmer pour autant qu'un transfert de savoir eut lieu des médecins orientaux aux médecins francs. Selon Conrad (1999), p. XLI-XLII, ces derniers s'étaient plutôt familiarisés avec la médecine galénique en Italie du Sud.

INDEX

Mots-clés : croisade, médecine, transfert culturel, dimmīs

Schlüsselwörter : Kreuzzug, Medizin, Kulturtransfer, dimmīs

\section{AUTEURS}

\section{JOHANNES PAHLITZSCH}

Johannes Pahlitzsch est professeur d'histoire byzantine à l'Université de Mayence. Pour plus d'informations, voir la notice suivante. 